\title{
Almost Budget-balanced Mechanisms for Allocation of Divisible Resources
}

\author{
Anil Kumar Chorppath and Srikrishna Bhashyam \\ Dept. of Elec. Engg. \\ Indian Institute of Technology Madras \\ Chennai 600036, India \\ ee07s018@smail.iitm.ac.in, skrishna@iitm.ac.in
}

\author{
Rajesh Sundaresan \\ Dept. of ECE \\ Indian Institute of Science \\ Bangalore 560012, India \\ rajeshs@ece.iisc.ernet.in
}

\begin{abstract}
Mechanisms for allocation of one or many goods to a number of agents are proposed in this paper. The goal is to propose mechanisms in a general setting where the goods might be divisible or indivisible. A mechanism in the Groves class that is dominant strategy incentive compatible and almost budget balanced is obtained. In the case of one or more indivisible goods, the proposed mechanisms fall back to the mechanisms proposed independently by Moulin and by Guo \& Conitzer. The proposed mechanisms are characterised by a linear redistribution or rebate function included in the payments. The proposed worst case optimal mechanism minimises the worst case ratio of budget surplus to efficient surplus. An optimal-in-expectation mechanism that minimizes the ratio of expected budget surplus to expected efficient surplus is also proposed and compared with the worst case optimal mechanism. Numerical solutions for the coefficients of linear rebate function, worst case efficiency loss, and expected efficiency loss are obtained. An example motivating the extension to the divisible case is resource allocation in the uplink of a wireless communication system. This is briefly discussed.
\end{abstract}

\section{INTRODUCTION}

Efficient resource allocation over a network often requires decision making based on knowledge of private information held by end agents. Strategic agents may misrepresent their privately held information in the interest of maximising their own benefit at the expense of overall system efficiency [1]. A socially optimal allocation should maximise efficiency (allocate so as maximise sum of all agents' utilities). In the Groves class of mechanisms [2], a social planner knows the valuation functions of the agents, allocates the goods (divisible or indivisible), and collects payments from agents. The payments affect the utilities of the agents and are constructed so that it is in the best interest of agents to reveal truthfully their privately held information. These mechanisms achieve allocative efficiency and are strategy-proof. The most celebrated mechanism in this class is the Vickrey-Clarke-Groves (VCG) mechanism (see [3]-[4]). The VCG mechanism maximises the total payments from the agents to the social planner. While this is indeed of interest in situations where an auctioneer sells his goods to agents, our interest is in scenarios where the resources have no owner and the social planner unlike the auctioneer desires no surplus (budget-balance). The wellknown Green-Laffont impossibility theorem [5], however, says

\footnotetext{
${ }^{0}$ This work is supported by Department of Science and Technology, Government of India.
}

that there is no mechanism in a quasi-linear environment that is strategy-proof, achieves allocative efficiency, and is budget-balanced. Moulin [6] and Guo \& Conitzer [7] proposed mechanisms within the Groves class for allocation of one or more homogeneous indivisible goods. Their mechanisms are almost budget-balanced. The purpose of this paper is to extend their mechanisms to the more general situation when goods are divisible.

Our interest in the above problem stemmed from a resource allocation problem on the uplink multiple access channel [8]. A base station has one or more channels that it can allocate to associated mobiles. Mobiles with larger residual packet queue lengths value the channel more than those with smaller queue lengths. However, queue lengths information reside privately with mobiles and strategic behaviour could jeopardise system efficiency. In this setting, mobiles are the agents and base station the social planner. The channels can be timeshared and allocations can be randomised so that we may take the goods (channels) to be perfectly divisible. In reality, no monetary exchange takes place in this setting. Price \& Javidi [8] consider modulating allocations on another independent link in lieu of payments. However, one may envisage reduced allocations on the uplink to adjust for payments. Therefore, any residual surplus is unutilised bandwidth and is undesirable. While this resource allocation problem motivated our study of almost budget-balanced, allocative efficient, strategy-proof mechanisms, our study has wider applicability.

Moulin's mechanism [6] for $p$ homogeneous indivisible goods ( $p<N$, the number of agents) may be interpreted as follows. The agents make the VCG payments. The social planner then provides rebates to the agents. The mechanism remains allocative efficient, individually rational, and strategyproof. Moreover, it minimises the worst (maximum) ratio of budget surplus to efficient surplus (sum of valuations) subject to the constraint that it is weakly budget balanced. Guo \& Conitzer[7] showed that the same mechanism maximises the worst-case (minimum) rebate redistribution fraction relative to the VCG payments. The optimal rebate for a particular agent is linear in the valuations of all other agents. Gujar \& Narahari [9] showed optimality of linear redistributions for $p$ heterogeneous indivisible goods when agents' valuations of each of the $p$ goods (each valuation is a $p$-tuple) have scaling based correlation. That is, there is a common vector $v$ such that 
any agent $i$ 's valuation vector is $\theta_{i} v$ where $\theta_{i}$ is a scalar private to agent $i$. We consider a more general setting than those considered by Moulin[6],Guo \& Conitzer [7] and Gujar \& Narahari [9]. The valuation function is of the form $v_{i}=\theta_{i} f($. where $f($.$) is a homogeneous function common to all agents.$ Also, the goods can be infinitely divisible.

The assumption that the valuation function is known to the social planner is often unrealistic. Reporting the entire valuation function is a considerable communication burden to the system [10]. Hence, mechanisms for allocation of a divisible good based on agents' scalar bids alone are of interest. If the mechanism allocates based only on the reported scalar bids, then dominant strategy implementation is not possible, and we ought to study Nash equilibria instead [11]. Yang and Hajek [12] proposed a VCG-Kelly mechanism by combining the one dimensional bid idea of Kelly, Maulloo \& Tan [13] with the VCG mechanism. The Nash Equilibrium Point (NEP) is shown to be unique and globally stable. The VCG-Kelly mechanism is studied in [12] for the case of a network rate allocation problem. Our proposed almost budget balanced mechanism can be extended to this network setting too.

The rest of the paper is organized as follows. Section II describes the system model and formulates the problem. Section III discusses the worst-case optimal mechanism. It also describes an approach to solve the optimisation problem under the linear redistribution setting. Section IV studies the optimalin-expectation mechanism. Section $\mathrm{V}$ describes simulation settings, assumptions, and presents simulation results. Section VI is a concluding summary of the paper.

\section{SYSTEM MODEL AND PROBLEM FORMULATION}

There is a set $S=\{1,2, \ldots, N\}$ of $N$ agents. There is one object which may be divisible or indivisible and it is to be allocated to these agents. The type of agent $i$, which is the private information of that agent, is $\theta_{i} \in R^{+}$. Two examples of type are: (1) the queue length of each agent in the uplink of a wireless communication system, and (2) the amount agents are ready to pay per unit time on a link in a communication network. The profile of types of all the agents is an $N \times 1$ vector $\theta \in \Theta$ given by $\left[\begin{array}{llll}\theta_{1} & \theta_{2} & \cdots & \theta_{N}\end{array}\right]^{T}$. Let $w \in \Theta$ be the profile of reported values of agents. A dominant strategy incentive compatible (DSIC) mechanism should make the dominant strategy of agents to reveal their type truthfully, i.e., $w=\theta$. Since we are considering mechanisms in the class of Groves mechanisms, which are DSIC, we may assume that all agents report their true types.

Let $a$ be an $N \times 1$ allocation vector taking values in $A$. The valuation of agent $i$ is taken to be a function of the allocation and the type of agent $i$ and is denoted by $v_{i}\left(a_{i}, \theta_{i}\right)$. Let $a^{*}$ be the socially optimal allocation, i.e.,

$$
a^{*}(\theta)=\arg \max _{a \in A} \sum_{i \in S} v_{i}\left(a_{i}, \theta_{i}\right) .
$$

Let $a_{-i} \in A_{-i}$ denote $N \times 1$ allocation vector when the $i^{t h}$ agent is out of contention, i.e., not considered for allocation.
So, $a_{-i, i}=0$. Let $a_{-i}^{*}$ be the optimal allocation when $i^{t h}$ agent is out of contention, i.e.,

$$
a_{-i}^{*}\left(\theta_{-i}\right)=\arg \max _{a_{-i} \in A_{-i}} \sum_{j \neq i} v_{j}\left(a_{-i, j}, \theta_{j}\right) .
$$

Consider the class of Groves mechanisms with rebate functions [2]. The payment $p_{i}(\theta)$ for the $i^{t h}$ agent under this mechanism is as follows:

$$
\begin{aligned}
p_{i}(\theta)= & \left.\sum_{j \neq i} v_{j}\left(a_{-i, j}^{*}\left(\theta_{-i}\right), \theta_{j}\right)\right) \\
& -\sum_{j \neq i} v_{j}\left(a_{j}^{*}(\theta), \theta_{j}\right)-r_{i}\left(\theta_{-i}\right),
\end{aligned}
$$

where the first two terms correspond to the VCG mechanism payment, and $r_{i}\left(\theta_{-i}\right)$ is the rebate for agent $i$. The rebate function essentially redistributes some of the VCG payment back to the agents. Note that the rebate is only a function of the types of other agents. The VCG payment for the $i^{\text {th }}$ agent is the difference in the sum of valuations of the other agents caused by the addition of the $i^{t h}$ agent to the system.

The payments are restricted to a class that satisfy following constraints.

1) Feasibility (F) or Weak Budget Balanced: This property ensures that the mechanism need not be subsidized by external supply of money. There is a net payment (budget surplus) from the agents to the mechanism.

$$
\sum_{i \in S} p_{i}(\theta) \geq 0 \text {. }
$$

Substitution of equation (1) in equation (2) yields

$$
\begin{aligned}
\sum_{i \in S} r_{i}\left(\theta_{-i}\right) \leq & \sum_{i \in S} \sum_{j \neq i} v_{j}\left(a_{-i, j}^{*}\left(\theta_{-i}\right), \theta_{j}\right) \\
& -(N-1) \sum_{i \in S} v_{i}\left(a_{i}^{*}(\theta), \theta_{i}\right) .
\end{aligned}
$$

Let the right hand side in equation (3) be denoted as $p_{V C G}(\theta)$.

2) Individual Rationality (or) Voluntary Participation (VP): This property ensures that the utility of all agents should be greater than or equal to the utility they would get by dropping out of the mechanism. The utility agents would get by not participating is usually taken to be zero. Thus

$$
v_{i}\left(a_{i}^{*}(\theta), \theta_{i}\right)-p_{i}(\theta) \geq 0, \forall i \in N .
$$

Substitution of equation (1) yields

$$
\begin{aligned}
\sum_{j \in S} v_{j}\left(a_{j}^{*}(\theta), \theta_{j}\right)- & \sum_{j \neq i} v_{j}\left(a_{-i, j}^{*}\left(\theta_{-i}\right), \theta_{j}\right) \\
& +r_{i}\left(\theta_{-i}\right) \geq 0, \forall i \in N,
\end{aligned}
$$

or, equivalently

$$
\begin{aligned}
r_{i}\left(\theta_{-i}\right) \geq & \sum_{j \neq i} v_{j}\left(a_{-i, j}^{*}\left(\theta_{-i}\right), \theta_{j}\right) \\
& -\sum_{j \in S} v_{j}\left(a_{j}^{*}(\theta), \theta_{j}\right), \forall i \in N .
\end{aligned}
$$


Let the right hand side in equation (5) be denoted as $n_{i}(\theta)$.

Adding all the $N$ constraints in equation (5) and using equation (3), we get

$p_{V C G}(\theta)-\sum_{i \in S} v_{i}\left(a_{j}^{*}(\theta), \theta_{i}\right) \leq \sum_{i \in S} r_{i}\left(\theta_{-i}\right) \leq p_{V C G}(\theta)$.

3) Anonymity: The mechanism treats all the agents the same, i.e., if agents' valuations are permuted, then so are the rebates.

Now, the problem is to find a payment which satisfies the above constraints, from within the class of Groves mechanisms, such that the resulting mechanism is almost budget balanced. We now make this precise.

\section{WORST-CASE OPTIMAL MECHANISM}

Moulin proposed a mechanism in [6] that minimizes the worst case efficiency loss. Let the efficient surplus be $\sigma_{v}(\theta)=$ $\sum_{i \in S} v_{i}\left(a_{j}^{*}(\theta), \theta_{i}\right)$. The worst case efficiency loss is the maximum ratio of budget surplus to the efficient surplus over all possible $\theta$, given by

$$
L(N)=\max _{\theta} \frac{\sum_{i} p_{i}(\theta)}{\sigma_{v}(\theta)} .
$$

The problem is to find a mechanism defined by the payments that minimises $L(N)$ subject to the constraints given in equations (3) and (5), i.e.,

$$
\min _{p(\theta)} \max _{\theta} \frac{\sum_{i} p_{i}(\theta)}{\sigma_{v}(\theta)}
$$

or, equivalently

$$
\min _{r} \max _{\theta} \frac{p_{V C G}(\theta)-\sum_{i} r_{i}\left(\theta_{-i}\right)}{\sigma_{v}(\theta)} .
$$

This optimal mechanism has been derived in [6] for the case when the goods are indivisible. The same mechanism has also been independently found in [7] using only linear redistribution schemes. In this paper, we propose a solution for the case when the goods are divisible.

\section{A. Linear redistribution mechanism}

We restrict attention to the following form of linear redistribution schemes. Let $\bar{v}\left(a_{-i}, \theta_{-i}\right)$ denote the vector of valuations of all agents except the $i^{\text {th }}$ agent when allocation is $a_{-i}$ and types are $\theta_{-i}$. It is a column vector with $N-1$ elements which are ordered in ascending order of agent index. Define

$$
\bar{m}_{i}\left(\theta_{-i}\right)=\sum_{a_{-i} \in A_{-i}} \bar{v}\left(a_{-i}, \theta_{-i}\right)
$$

to be the column vector whose elements are sum of valuations obtained from all possible allocations without the $i^{t h}$ agent. The summation in the above equation becomes an integral in the divisible case and is given by

$$
\bar{m}_{i}\left(\theta_{-i}\right)=\int_{a_{-i} \in A_{-i}} \bar{v}\left(a_{-i}, \theta_{-i}\right) d a_{-i} .
$$

Let $m_{i}\left(\theta_{-i}\right)$ be made of components of $\bar{m}_{i}\left(\theta_{-i}\right)$ ordered in descending order of values. The rebate is a linear combination of the $m_{i}\left(\theta_{-i}\right)$ and is given by

$$
r_{i}\left(\theta_{-i}\right)=c^{t} \cdot m_{i}\left(\theta_{-i}\right)
$$

where $c=\left[c_{1}, c_{2}, \ldots, c_{N-1}\right]^{t}$ is coefficient vector of $N-1$ elements.

We may thus state the optimisation problem for the linear redistribution scheme using equations (3), (5), (7) and (9) as:

Problem (P):

$$
\min _{c} \max _{\theta} \frac{p_{V C G}(\theta)-\sum_{i} c^{t} \cdot m_{i}\left(\theta_{-i}\right)}{\sigma_{v}(\theta)}=\min _{c} \Phi(c)
$$

subject to

1) $\sum_{i} c^{t} \cdot m_{i}\left(\theta_{-i}\right) \leq p_{V C G}(\theta), \forall \theta$

2) $c^{t} \cdot m_{i}\left(\theta_{-i}\right) \geq n_{i}(\theta), \forall i \in N, \forall \theta$.

The objective function $\Phi$ is a convex function since it is a maximum over a family of affine functions with coefficients parametrized by $\theta \in \Theta$. In constraint 1 , let $C_{1}(\theta)$ be a set of feasible coefficients for a given value of $\theta$. This defines a half plane which is a convex set. Let $C_{1}=\bigcap_{\theta} C_{1}(\theta)$. The intersection of half planes is again convex. Thus $C_{1}$ is a convex set. In constraint 2 , let $C_{2}(\theta)$ be a set of feasible coefficients for given value of $\theta$. Let $C_{2}=\bigcap_{\theta} C_{2}(\theta)$. Similarly, $C_{2}$ is also a convex set. Let $C=C_{1} \cap C_{2}$ be the set of coefficients which satisfies both constraints. Then, $C$ is also a convex set. Thus Problem $\mathbf{P}$ is a convex optimisation problem.

The problem $\mathbf{P}$ can be rewritten as a new minimisation problem by introducing one additional constraint as,

$$
\min _{c, L(N)} L(N)
$$

subject to

1) $\sum_{i} c^{t} \cdot m_{i}\left(\theta_{-i}\right) \leq p_{V C G}(\theta), \forall \theta$

2) $c^{t} \cdot m_{i}\left(\theta_{-i}\right) \geq n_{i}(\theta), \forall i \in N, \forall \theta$,

3) An additional constraint:

$$
\frac{p_{V C G}(\theta)-\sum_{i} c^{t} \cdot m_{i}\left(\theta_{-i}\right)}{\sigma_{v}(\theta)} \leq L(N), \forall \theta,
$$

or, equivalently

$$
\sum_{i} c^{t} \cdot m_{i}\left(\theta_{-i}\right)+L(N) \sigma_{v}(\theta) \geq p_{V C G}(\theta), \quad \forall \theta .
$$

We next restrict attention to valuation of the $i^{\text {th }}$ agent of the form $v_{i}=\theta_{i} f\left(a_{i}\right)$, where $f(\cdot)$ is a differentiable nondecreasing concave function over $\Re^{+}$with $f(0)=0$.

The case of allocation of $p$ homogeneous indivisible goods to $N$ agents as considered by Moulin [6] and Guo \& Conitzer [7] is a special case of the above valuation function and the allocation set. Indeed, let $f(x)=x \forall x \in \Re^{+}$and $A$ is such that $\sum_{i} a_{i}=1$. In this case, the above optimisation problem can be reduced to the linear program solved by them. Another interesting case is when $f(\cdot)$ is linear in $\Re^{+}$and $A$ is such that $\sum_{i} a_{i}=p$. Then all the $p$ goods will be allocated to the agent having the highest value of $\theta$. This case will essentially 
reduce to the allocation of one indivisible good to $N$ agents as solved in [6] and [7].

Consider now the allocation of a single divisible good to $N$ agents. The optimal allocation solves

$$
a^{*}(\theta)=\arg \max _{a \in A} \sum_{i} \theta_{i} f\left(a_{i}\right) .
$$

From equation (8), the $j^{\text {th }}$ term of $\bar{m}_{1}\left(\theta_{-1}\right)$ will be

$$
\int_{A_{-1}} \theta_{j} f\left(a_{j}\right) d a_{-1} \propto \theta_{j} \int_{A_{j}} f\left(a_{j}\right) \cdot d a_{j} \propto \theta_{j} .
$$

Due to symmetry, we have

$$
\bar{m}_{i}\left(\theta_{-i}\right)=\left(\begin{array}{llllll}
\theta_{1} & \theta_{2} & \cdots & \theta_{i-1} & \theta_{i+1} & \cdots \theta_{N}
\end{array}\right)^{t} .
$$

We now assume without loss of generality that $\theta_{1} \geq \theta_{2} \geq \cdots \geq \theta_{N}$, and therefore $m_{i}\left(\theta_{-i}\right)=$

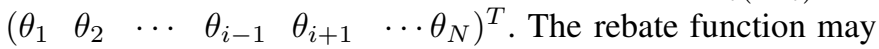
now be written as

$$
r_{i}\left(\theta_{-i}\right)=c^{t} *\left(\begin{array}{c}
\theta_{1} \\
\theta_{2} \\
\vdots \\
\theta_{i-1} \\
\theta_{i+1} \\
\vdots \\
\theta_{N}
\end{array}\right)
$$

Using equation (12), problem $\mathbf{P}$ becomes:

$$
\min _{c, L(N)} L(N)
$$

subject to,
1) $\sum_{i=1}^{N-1} c_{i}\left(i \theta_{i+1}+(N-i) \theta_{i}\right) \leq p_{V C G}(\theta), \forall \theta$,
2) $r_{i}\left(\theta_{-i}\right) \geq n_{i}(\theta), \forall \theta, \forall i \in N$,
3) $\sum_{i=1}^{N-1} c_{i}\left(i \theta_{i+1}+(N-i) \theta_{i}\right)+L(N) \sigma_{v}(\theta) \geq p_{V C G}(\theta), \forall \theta$.

Let $e_{k}=(1, \ldots 1,0, \ldots, 0)$ with $k$ consecutive 1 's. Putting $\theta=e_{1}$, we get $p_{V C G}(\theta)=0$ and $n_{i}(\theta)=0 \forall i \geq 2$. Therefore, using constraint 1 , we get $(N-1) c_{1} \leq 0$. Using constraint 2 , we get $r_{2}\left(\theta_{-2}\right)=c_{1} \geq 0$. Thus $c_{1}=0$. The following lemma is useful in simplifying constraint 2 .

Lemma 1: The following system of inequalities are equivalent.

(a) $r_{i}\left(\theta_{-i}\right) \geq n_{i}(\theta), \forall \theta, \forall i \in N$

(b) $\sum_{i=2}^{k} c_{i} \geq 0, \forall k=2,3, \ldots, N-1$.

Proof: (a) $\Rightarrow$ (b): The definition of $n_{i}(\theta)$ in the right-hand side of equation (5) and the form of the valuation function $v_{i}\left(a_{i}, \theta_{i}\right)=\theta_{i} f\left(a_{i}\right)$ yields

$$
n_{i}(\theta)=\sum_{j \neq i}^{N} \theta_{j} f\left(a_{-i, j}^{*}\left(\theta_{-i}\right)\right)-\sum_{j=1}^{N} \theta_{j} f\left(a_{j}^{*}(\theta)\right) \leq 0 .
$$

The last inequality is because $a_{-i}^{*}$ is inefficient allocation when compared with $a^{*}(\theta)$ when all the $N$ agents are active.

Next the rebates are given by,

$$
r_{i}\left(\theta_{-i}\right)=c_{2} \theta_{2}+\cdots+c_{i-1} \theta_{i-1}+c_{i} \theta_{i+1}+\cdots+c_{N-1} \theta_{N} .
$$

Consider $\theta=e_{k}$ for $k=2,3, \ldots, N-1$. We then have

$$
r_{k+1}\left(\theta_{-(k+1)}\right)=\sum_{i=2}^{k} c_{i}
$$

and

$$
\begin{gathered}
n_{k+1}(\theta)=\sum_{j \neq k+1}^{N} \theta_{j} f\left(a_{-(k+1) j}^{*}\left(\theta_{-(k+1)}\right)\right)-\sum_{j=1}^{N} \theta_{j} f\left(a_{j}^{*}(\theta)\right) \\
=\sum_{j=1}^{k} f\left(a_{-(k+1) j}^{*}\left(\theta_{-(k+1)}\right)\right)-\sum_{j=1}^{k} f\left(a_{j}^{*}(\theta)\right) \\
=0
\end{gathered}
$$

because $a_{-(k+1)}^{*}\left(\theta_{-(k+1)}\right)=a^{*}(\theta)$ as a consequence of the fact that $\theta_{-(k+1)}=\theta=e_{k}$.

Putting these together, $\sum_{i=2}^{k} c_{i} \geq 0 \forall k=2,3, \ldots, N-1$.

(b) $\Rightarrow$ (a): From Lemma 1 proved by Guo \& Conitzer in [7], if $\sum_{i=2}^{k} c_{i} \geq 0$ for all $k=2,3, \ldots, N-1$ then, $c_{2} \theta_{2}+$ $\cdots+c_{i-1} \theta_{i-1}+c_{i} \theta_{i+1}+\cdots+c_{N-1} \theta_{N} \geq 0$ for all $\theta_{1} \geq$ $\theta_{2} \geq \theta_{3} \cdots \geq \theta_{N}$. Consequently $r_{i}\left(\theta_{-i}\right) \geq 0 \forall i \in N$ and the reverse implication follows from equation 14 . This proves the lemma.

Problem $\mathbf{P}$ therefore becomes

$$
\min _{c_{2}, \ldots, c_{N-1}, L(N)} L(N)
$$

subject to

1) $\sum_{i=2}^{N-1} c_{i}\left(i \theta_{i+1}+(N-i) \theta_{i}\right) \leq p_{V C G}(\theta), \forall \theta$,
2) $\sum_{\substack{i=2 \\ k}} c_{i} \geq 0, \forall k=2,3, \ldots, N-1$,
3) $\sum_{i=2}^{N-1} c_{i}\left(i \theta_{i+1}+(N-i) \theta_{i}\right)+L(N) \sigma_{v}(\theta) \geq p_{V C G}(\theta), \forall \theta$.

The above convex optimisation problem is numerically solved to obtain the optimal coefficients of the linear rebate function. The simulation results are studied in section 5 .

\section{OPTIMAL-IN-EXPECTATION MECHANISM}

In some scenarios, the worst case $\theta$ profiles may not occur very often. One may wish to minimize the efficiency loss in an expected sense. Thus, we design another mechanism in the class of Groves mechanisms which is optimal in expectation. The prior distribution over the agents' types are assumed to be 
known and the objective is to minimise the expected efficiency loss given by

$$
\frac{E\left[p_{V C G}(\theta)-\sum_{i=1}^{N} r_{i}\left(\theta_{-i}\right)\right]}{E\left[\sum_{i \in S} v_{i}\left(a_{i}^{*}(\theta), \theta_{i}\right)\right]},
$$

subject to the same constraints (F and VP) as in the worst case problem. By using the same form of linear rebate function as proposed above, the objective function becomes (for all $\theta_{1} \geq \theta_{2} \geq \cdots \geq \theta_{N-1} \geq \theta_{N}$ and variables $\left.c_{2}, \cdots, c_{N-1}\right)$

$$
\frac{E\left[p_{V C G}(\theta)\right]-E\left[\sum_{i=2}^{N-1} c_{i}\left(i \theta_{i+1}+(N-i) \theta_{i}\right)\right]}{E\left[\sigma_{v}(\theta)\right]} .
$$

Given prior distributions, the quantities $E\left[\theta_{i}\right], E\left[\sigma_{v}(\theta)\right]$ and $E\left[p_{V C G}(\theta)\right]$ are constants. Thus the problem becomes,

$$
\max _{c_{2}, \ldots, c_{N-1}} \sum_{i=2}^{N-1} c_{i}\left(i E\left[\theta_{i+1}\right]+(N-i) E\left[\theta_{i}\right]\right),
$$

subject to,

1) $\sum_{i=2}^{N-1} c_{i}\left(i \theta_{i+1}+(N-i) \theta_{i}\right) \leq p_{V C G}(\theta), \forall \theta$,

2) $\sum_{i=2}^{k} c_{i} \geq 0, \forall k=2,3, \ldots, N-1$.

The above convex optimisation problem is also numerically solved to obtain the optimal coefficients of the linear rebate function.

\section{Simulation Setup AND Results}

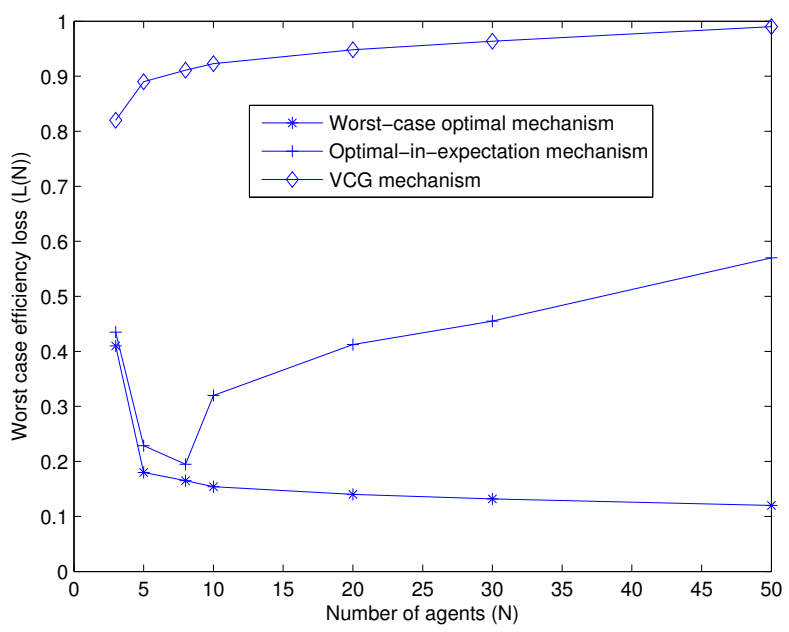

Fig. 1. Worst efficiency loss of worst case optimal and VCG mechanisms

In the two convex optimisation problems above, some constraints are a function of $\theta \in \Theta=[0,1]^{N}$. We thus have a continuum of half-space constraints whose intersection is $C$. Guo \& Conitzer [7] proved that the constraints obtained with $\theta$ profiles as $e_{k}=(1,1, \ldots 1,0, \ldots 0)$ with $k 1$ 's for $k=0,1, \ldots, N$ are enough to specify the feasible region in the case of indivisible goods. We also start with these $\theta$ profiles. However, it was observed from simulations that these $\theta$ profiles are not enough to characterise the feasible region for the divisible goods case. Therefore, additional constraints were obtained by sampling 50 random values of $\theta$ uniformly on $\Theta$. This yielded an approximation $\hat{C}$ of $C$ that contained $C$. The coefficients of the rebate function are now obtained by numerically solving the minimisation over $\hat{C}$. It was observed that $\hat{C}$ approximated the actual feasible region well because the coefficients generated using this feasible region did not violate the constraints for $10000 \theta$ 's sampled uniformly on $\Theta$.

Worst case efficiency loss of our proposed mechanism is obtained by simulation for the valuation function $v_{i}=$ $\theta_{i} \log \left(1+a_{i}\right)$. The set $A$ is defined by the set of all allocation vectors that satisfy $\sum_{i} a_{i}=1 . \theta$ is uniform on $\Theta$. The optimal allocation satisfies,

$$
a_{i}^{*}(\theta)=\left[\frac{\theta_{i}}{\lambda(\theta)}-1\right]_{+} .
$$

The worst case efficiency loss $(L(N))$ and coefficients $c_{2}, c_{3}, \cdots, c_{N-1}$ are obtained by solving the optimisation problem numerically. For the optimal-in-expectation mechanism, the feasibility region is obtained in an analogous fashion with the modified objective function. Since $\theta$ is uniformly distributed on $\Theta$ and then subsequently ordered, the ordered quantities satisfy $E\left[\theta_{i}\right]=\frac{N-i+1}{N+1}, i=1,2, \cdots, N$. The coefficients $c_{2}, c_{3}, \cdots, c_{N-1}$ are obtained by solving the optimisation problem on the approximate feasible region. Efficiency losses for $20000 \theta$ profiles are calculated under these coefficients. The expected efficiency loss is obtained by taking the sample mean. The worst efficiency loss for optimal-in-expectation mechanism is obtained by calculating the efficiency losses for the $e_{k}$ profiles and 20000 uniformly sampled $\theta$ profiles followed by an identification of the worst case among them.

The worst case optimal mechanism is compared with VCG mechanism and optimal-in-expectation mechanism in Figure 1 for worst case efficiency loss. It is observed that as number of agents increases the worst case efficiency loss reduces for the worst case optimal mechanism. On the other hand, the worst case efficiency loss converges to 1 for the VCG mechanism. As expected, the optimal-in-expectation performs poorly in the worst case sense compared to worst case optimal mechanism, especially for large number of agents. In Figure 2 , the expected efficiency loss of the optimal-in-expectation mechanism is compared with the worst case optimal and VCG mechanisms. It can be seen from Figure 2 that the optimal in expectation mechanism outperforms the other two mechanisms in the expectation sense. The expected efficiency loss of the optimal-in-expectation and worst case mechanisms reduce as the number of agents increases. On the other hand, the expected efficiency loss of the VCG mechanism increases 


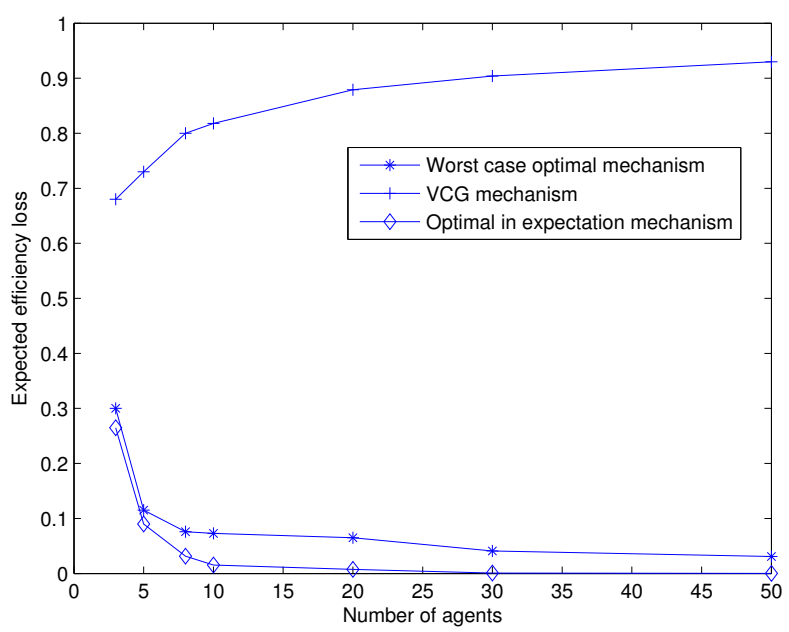

Fig. 2. Expected efficiency loss of optimal-in-expectation, worst case optimal and VCG mechanisms

as the number of agents increase.

\section{CONCLUSiOnS}

We proposed a mechanism in the class of Groves mechanisms that is almost budget-balanced as it minimizes the worst case efficiency loss. The proposed mechanism is feasible and has voluntary participation and anonymity properties. The mechanism is applicable to allocation of divisible or indivisible goods and simplifies to the mechanism proposed by Moulin [6] and Guo \& Conitzer [7] for the indivisible goods case. A mechanism that is optimal-in-expectation is also proposed. This is an extension of the Guo \& Conitzer mechanism in [14] for one (or) more indivisible goods to the divisible goods case. The proposed mechanisms are compared with each other and with the VCG mechanism to show the improvement in efficiency.

The mechanisms we proposed can be extended to a case where the valuation functions are private information to agents and unknown to the central planner but can be parametrised by a scalar value. Yang \& Hajek [12] had proposed a VCGKelly mechanism in a network resource allocation setting that achieves an efficient Nash equilibrium implementation. Our proposed mechanisms can be extended to this setting as well. The mechanisms with better redistribution by allowing the allocation to be inefficient can be also looked in divisible setting. A linear rebate function is used in the proposed mechanisms. The optimality or otherwise of the proposed linear rebate mechanism is under study.

\section{REFERENCES}

[1] R. Johari and J. Tsitsiklis, "Efficiency loss in a network resource allocation game," Mathematics of Operations Research, vol. 29, no. 23, pp. 407-435, August 2004.

[2] T. Groves, "Incentives in teams," Econometrica, vol. 41, no. 4, pp. 617 631,1973

[3] W. Vickrey, "Counterspeculation, auctions and competitive sealed tenders," Journal of Finance, vol. 16, no. 1, pp. 8-37, 1961.
[4] E. Clarke, "Multipart pricing of public goods," Public Choice, vol. 2, pp. 19-33, 1971.

[5] A. Mas-Colell, M. Whinston, and J. Green, Microeconomic Theory. Oxford University Press, 1995.

[6] H. Moulin, "Almost budget-balanced VCG mechanisms to assign multiple objects," Journal of Economic theory, vol. 144, no. 1, pp. 96-119, January 2009.

[7] M. Guo and V. Conitzer, "Worst-case redistribution of VCG payments," in 8th ACM Conference on Electronic Commerce, San Diego, California, USA, June 2007, pp. 30-39.

[8] J. Price and T. Javidi, "Leveraging downlink for efficient uplink allocation in a single-hop wireless network," IEEE Tran. on Inform. Theory, vol. 53, no. 11, pp. 4330-4339, November 2007.

[9] S. Gujar and Y. Narahari, "Redistribution of VCG payments in assignment of heterogeneous objects," in 4th International Workshop on Internet and Network Economics(WINE), 2008, pp. 438-445.

[10] R. Johari and J. Tsitsiklis, "Communication requirements of VCGlike mechanisms in convex environments," in Allerton Conference on Communication, Control and Computing, 2005.

[11] S. Sanghavi and B. Hajek, "Optimal allocation of a divisible good to strategic buyers," in IEEE Conference on Decision and Control, vol. 3, December 2004, pp. 2748-2753.

[12] S. Yang and B. Hajek, "VCG-kelly mechanisms for allocation of divisible goods: Adapting VCG mechanisms to one-dimensional signals," IEEE Journal on Selected Areas in Communication, vol. 25, no. 6, pp. 1237-1243, August 2007

[13] F. P. Kelly, A. K. Maulloo, and D. Tan, "Rate control in communication networks: shadow prices, proportional fairness and stability," Journal of the Operational Research Society, vol. 49, pp. 237-252, 1998.

[14] M. Guo and V. Conitzer, "Optimal-in-expectation redistribution mechanisms," in International Conference on Autonomous Agents and MultiAgent Systems (AAMAS), Estoril, Portugal, 2008, pp. 1047-1054. 